\title{
EL USO DEL SMARTPHONE PARA LA GESTIÓN DEL TRABAJO COLABORATIVO EN ESTUDIANTES DE EDUCACIÓN SUPERIOR ARGENTINA DURANTE LA PANDEMIA DEL COVID-19
}

\author{
Francisco Javier Albarello* \\ Universidad del Salvador \\ albarello.francisco@usal.edu.ar \\ FRANCISCO HERNANDO ARRI** \\ Universidad del Salvador \\ farri@usal.edu.ar \\ ANA LAURA García LUnA*** \\ Universidad del Salvador \\ algaluna@usal.edu.ar
}

Recibido: 15/5/2021 Aceptado: 10/8/2021

doi: https://doi.org/10.26439/contratexto2021.n036.5195

RESUMEN. La situación de aislamiento social obligatorio debido a la pandemia global del COVID-19 ha afectado a todos los órdenes de la vida, y la educación no ha sido la excepción. No solo las universidades se han adaptado al nuevo panorama ofreciendo instancias de enseñanza virtual, sino que los estudiantes han desarrollado prácticas de estudio colaborativas valiéndose de los smartphones y otros dispositivos digitales. Este artículo presenta los resultados de una investigación cualitativa que incluyó entrevistas en profundidad a cuarenta y cinco estudiantes universitarios argentinos, con el objeto de indagar sobre los cambios que ha generado la cuarentena en las formas de estudio. Los hallazgos indican que los estudiantes desarrollan complejas estrategias de estudio colaborativo, en las que alternan entre textos, audios y otros materiales y aplicaciones

\footnotetext{
* Doctor en Comunicación Social por la Universidad Austral, Argentina (véase: https://orcid.org/0000-00016623-916X).

** Doctor en Comunicación Social por la Universidad Austral, Argentina (véase: https://orcid.org/0000-00028946-9870).

*** Magíster en Periodismo de Investigación por la Universidad del Salvador, Argentina (véase: https://orcid. org/0000-0001-8712-0176)
} 
que les permiten estudiar y compensar la ausencia física de sus compañeros a través del trabajo colaborativo mediado por las pantallas.

PALABRAS CLAVE: COVID-19 / estudiantes / smartphone / aprendizaje colaborativo / enseñanza superior

\section{THE USE OF THE SMARTPHONE FOR THE MANAGEMENT OF COLLABORATIVE WORK IN ARGENTINE HIGHER EDUCATION STUDENTS DURING THE COVID-19 PANDEMIC}

ABSTRACT. The situation of mandatory social isolation due to the global COVID-19 pandemic has affected all walks of life, and education has not been the exception. Not only have universities adapted to the new landscape by offering virtual teaching instances, but students have developed collaborative study practices using Smartphones and other digital devices. This article presents the results of a qualitative research that included in-depth interviews with 45 Argentine university students, to investigate the changes that the quarantine has generated in their ways of studying. The findings indicate that students develop complex collaborative study strategies, alternating between texts, audios, and other materials and applications that allow them to study and compensate for the physical absence of their classmates through collaborative work mediated by screens.

KEYWORDS: COVID-19 / students / smartphone / collaborative learning / higher level education

\section{O USO DO SMARTPHONE PARA A GESTÃO DO TRABALHO COLABORATIVO EM ESTUDANTES ARGENTINOS DO ENSINO SUPERIOR DURANTE A PANDEMIA DE COVID-19}

RESUMO. A situação de isolamento social obrigatório devido à pandemia global COVID19 afetou todas as esferas da vida, e a educação não foi a exceção. Não apenas as universidades se adaptaram ao novo cenário oferecendo instâncias virtuais de ensino, como também os alunos desenvolveram práticas de estudo colaborativo usando Smartphones e outros dispositivos digitais. Este artigo apresenta os resultados de uma pesquisa qualitativa que incluiu entrevistas em profundidade a 45 estudantes universitários argentinos, com o objetivo de investigar as mudanças que a quarentena gerou nas formas de estudo. Os resultados indicam que os alunos desenvolvem estratégias de estudo colaborativas complexas, nas quais alternam textos, áudios e outros materiais e aplicativos que permitem estudar e compensar a ausência física de seus colegas por meio de trabalhos colaborativos mediados por telas.

PALAVRAS-CHAVE: COVID-19 / estudantes / smartphone / aprendizagem colaborativa / ensino superior 


\section{INTRODUCCIÓN}

En los últimos años, la presencia de los dispositivos móviles inteligentes ha impactado y atravesado la vida social y sus prácticas. Gracias a su condición ubicua y transportable, el smartphone ha logrado formar parte fundamental del contexto general de la "sociedad red". En este sentido, las redes se presentan como una estructura que se organiza gracias a un nuevo entorno tecnológico-digital a partir de su flexibilidad, adaptabilidad y capacidad de supervivencia (Castells, 2009). Así, el objetivo de este artículo es explorar la utilización del smartphone para el trabajo académico colaborativo por parte de estudiantes de educación superior argentina durante el aislamiento social preventivo y obligatorio que suspendió las clases presenciales en el país a causa de la pandemia del COVID-19.

La proliferación de los dispositivos móviles ha transformado radicalmente el consumo de noticias, de productos y servicios, así como los procesos de enseñanza y de aprendizaje, dado que la vida social aparece atravesada por la intermediación del software (Manovich, 2013), las plataformas mediáticas (Fernández, 2018, 2021; Srnicek, 2018; Van Dijck, 2016) y las pantallas (Márquez, 2015, 2017).

En Argentina las cifras sobre el grado de penetración de los dispositivos móviles son elocuentes y pueden dar cuenta de la relevancia del problema que se plantea. En este sentido, el Instituto Nacional de Estadísticas y Censos (INDEC) ha documentado, a través de la Encuesta Permanente de Hogares en el cuarto trimestre del 2019, que el $84,3 \%$ de la población encuestada en 31 aglomerados urbanos del país tiene acceso a un smartphone. La cifra desciende al $41,4 \%$ cuando los encuestados responden si tienen computadora. Si se observan los datos estratificados por edad de uso, puede notarse que el $95,9 \%$ de las personas de entre 18 y 29 años utiliza un dispositivo móvil para comunicarse, mientras que si esa información se procesa tomando en cuenta el nivel educativo, el 98,5\% de los encuestados que está cursando una carrera de nivel terciario o universitario usa un teléfono móvil para comunicarse. Este porcentaje es el más alto respecto de otros grupos (como, por ejemplo, los que tienen menor nivel de formación académica).

\section{La evolución de los medios hacia el aprendizaje móvil}

Desde el punto de vista de la ecología de los medios, teoría desarrollada principalmente por McLuhan (1994) y Postman (1985), los medios de comunicación crean a su alrededor, en entorno sensorial, un ambiente que modifica los patrones de percepción de sus usuarios y estos cambios pasan desapercibidos. Esto ha sido definido por Scolari (2015) como la dimensión ambiental de la ecología de los medios. Asimismo, estos autores sostienen que los medios conforman un ecosistema complejo, en donde un medio no puede ser comprendido sino en interacción con otros medios (McLuhan, 1994). Desde la perspectiva 
de la mediamorfosis (Fidler, 1998), los medios evolucionan en forma convergente, y cuando aparece un nuevo medio, al principio toma prestadas las características principales del medio anterior, para luego diferenciarse y madurar. Jenkins (2008) también se refiere a este fenómeno cuando habla de "convergencia mediática" y sostiene que los medios interactuarán de maneras complejas e imprevisibles, y las audiencias asumirán un rol clave en esa evolución. Asimismo, para Scolari (2018), los medios evolucionan - coevolucionan- en relación con los otros medios y también en relación con los usuarios, a través del espacio interactivo que representa la interfaz. La interfaz puede entenderse como una "gramática de la interacción" entre el usuario y la computadora (Scolari, 2004), pero más adelante este autor lleva el concepto de interfaz a una diversidad de dispositivos y prácticas sociales en las que se desarrolla un diálogo entre el usuario y el sistema, diálogo a través del cual se produce una negociación de sentido, porque la interfaz siempre admite usos desviados y consecuencias no previstas por el diseñador (Scolari, 2018). Desde una perspectiva similar, Manovich (2013) habla del software como interfaz, puesto que en nuestra vida cotidiana cada vez más nuestras acciones están mediadas por algún tipo de programa informático que funciona como interfaz con el mundo exterior, con nuestras relaciones sociales y con nosotros mismos.

Manovich (2013) sostiene la idea de la computadora y del software como un nuevo medio de comunicación, más precisamente un metamedium que incluye a los medios anteriores, a los cuales, por un lado, los emula o imita gracias a la digitalización - por ejemplo, las simulaciones de interfaces de lectura de libros o diarios, los reproductores de música o de video-y, por otro lado, los remedia o reformula (Bolter, 2001) formando nuevos híbridos (Manovich, 2013). La computadora como metamedium, entonces, no solo se limita a emular a los medios anteriores, sino que además ofrece una plataforma de creación de nuevos medios debido a las herramientas que brinda a sus usuarios, convirtiéndose de ese modo en un editor de medios personal (Manovich, 2013).

Las apropiaciones que hacen los usuarios de las herramientas digitales que les ofrece la computadora como metamedium adquieren una relevancia central en lo relacionado con el aprendizaje. Precisamente, una de las características que tienen estas "tecnologías relacionales" (Aguado et al., 2013) es el aspecto colaborativo. El aprendizaje colaborativo resalta la importancia de la interacción social en el desarrollo del conocimiento y del pensamiento (Bruning et al., 2004).

Asimismo, la relación entre aprendizaje colaborativo y tecnología digital es estrecha, a tal punto que una de las áreas de investigación derivada de la colaboración asistida por computadora - computer supported collaboration (CSC) - es precisamente el aprendizaje colaborativo asistido por computadora - computer supported collaborative learning (CSCL) -, que tiene como objetivo mejorar el proceso de aprendizaje y los resultados académicos de los estudiantes con la ayuda de la tecnología (Cote-Parra, 2015). 
Otro concepto clave para comprender la potencialidad de las herramientas digitales al servicio del aprendizaje es la multimodalidad, concepto desarrollado por Kress (2005) que "alude a la diversidad de lenguajes que incluyen actualmente gran parte de los mensajes de circulación social" (Albarello y Kelly, 2019, p. 3). Dada la significación amplia que presenta el término lenguaje, Kress (2005) prefiere hablar de "modo"; entonces, multimodales son aquellos mensajes que combinan diferentes modos en su construcción. A partir de la convivencia de estos diferentes modos de representación, plantea la necesidad de construir una nueva teoría sobre el texto, "una teoría multimodal del alfabetismo" (Kress, 2005, p. 50). Desde esta perspectiva, utilizará el término texto "para cualquier caso de comunicación que se produzca en cualquier modo, o en cualquier combinación de modos" (Kress, 2005, p. 67).

En este sentido, si comprendemos a la multimodalidad en el contexto actual y desde la perspectiva de la ecología de los medios y de la convergencia digital, podemos asumir que la digitalización del texto, en sus más variables formas, no hizo otra cosa que llevar esta definición de texto a nuevas fronteras, donde se mezclan formatos, lenguajes y géneros en un proceso de creciente hibridación (Scolari, 2012), y en la cual los usuarios - los estudiantes, en este caso- asumen un rol importante cuando intervienen, comparten y remezclan el contenido.

Las tecnologías digitales, en general, y las móviles, en particular, han provocado ciertas transformaciones respecto de las metodologías tradicionales de enseñanza que se venían utilizando. En el nivel superior, hay un doble desafío pedagógico: por un lado, los dispositivos se presentan como herramientas de interacción. Por el otro, está la cuestión de la pérdida de atención y distracción por parte de los estudiantes (Silva Calpa y Martínez Delgado, 2017). De acuerdo con la visión de estos autores, el teléfono móvil inteligente ha permitido adaptar a un modelo ubicuo los procesos de enseñanza y aprendizaje. Por su parte, Flórez Buitrago et al. (2016) aseguran que los smartphones en entornos de educación superior facilitan la inclusión social, dado que los estudiantes prestan atención a la creatividad, la solución de problemas, la experimentación y la comunicación, entre otros factores.

Desde esta perspectiva, el aprendizaje móvil como propuesta utiliza los dispositivos como herramientas educativas; además:

se basa en premisas vinculadas con la economía de los dispositivos, la extensión de la disponibilidad de smartphones y tablets en la comunidad estudiantil, el dominio y la motivación que genera su uso entre el alumnado y la gran variedad de aplicaciones que se generan día a día. (Morán, 2021, p. 100)

Según un estudio de Dafonte-Gómez et al. (2021), el m-learning permite diseñar actividades formativas que facilitan la interconexión de alumnado, docentes y materiales sin limitaciones geográficas o cronológicas, fomenta el trabajo colaborativo y 
hace posible el aprendizaje ubicuo y en movimiento. En tanto, de acuerdo con la bibliografía analizada por Baert et al. (2020), existen dos visiones contrapuestas respecto de los efectos que tiene el uso del smartphone sobre la performance de los estudiantes: por un lado, están quienes dicen que el teléfono inteligente puede mejorar la eficiencia de las actividades académicas al permitir que los estudiantes busquen continuamente información y al facilitar el trabajo en equipo; y, por otro, están quienes afirman que los estudiantes ven al smartphone principalmente como una fuente de entretenimiento antes que como una herramienta para estudiar (Baert et al., 2020). Para graficar esta controversia, podemos destacar que en Argentina rigió hasta el 2016 una resolución de la Dirección General de Escuelas de la Provincia de Buenos Aires que prohibía el uso de teléfonos móviles en las escuelas' ${ }^{1}$.

Como veremos a continuación, el uso de dispositivos móviles para el aprendizaje no ha hecho más que crecer en el contexto del aislamiento social originado por la pandemia global del COVID-19, ya que en muchos casos el teléfono celular se ha convertido en un aliado importante para garantizar la continuidad pedagógica.

\section{El aprendizaje en tiempos de pandemia}

El uso del smartphone se ha acrecentado significativamente durante la "nueva normalidad" producida por la pandemia global del COVID-19 (Aufa et al., 2020). Un análisis bibliométrico realizado por Mustapha et al. (2021) en relación con la efectividad de la tecnología digital en la educación durante la pandemia destaca que la educación digital instaura un ambiente de aprendizaje mezclado - blended-, en el cual los teléfonos móviles juegan un rol importante. Desde una perspectiva cuantitativa, uno de los métodos más utilizados para analizar la adopción y el uso de los teléfonos móviles en las aulas universitarias es el modelo de aceptación de tecnología (Davis et al., 1989). Desde esta perspectiva, algunos estudios (Chuchu y Ndoro, 2019; Rojas-Osorio y Álvarez-Risco, 2019) indican que los factores que pueden predecir la adopción del teléfono móvil por parte de los estudiantes universitarios son los siguientes: la utilidad percibida, la facilidad de uso percibida, las actitudes hacia una aplicación móvil y la intención de utilizar una aplicación móvil.

1 La Resolución 1728, dictada por la Dirección General de Escuelas de la Provincia de Buenos Aires en junio del 2006, prohibía en todo el sistema educativo la utilización de celulares dentro del ámbito escolar y en el horario de clase. Esta resolución fue derogada en octubre del 2016 por el mismo organismo. El argumento del entonces ministro de Educación de la provincia, Alejandro Finocchiaro, fue que, a diferencia de lo que sucedía antes, cuando el teléfono solo se usaba para llamadas y era disruptivo en el aula, "hoy los celulares permiten otras cosas y queremos darle la posibilidad a docentes y alumnos [de] que utilicen cualquier dispositivo tecnológico siempre que esté incluido en el proyecto educativo institucional de cada escuela" (Derogan la resolución que prohibía los celulares para uso pedagógico, 2016). 
Otra de las teorías que se reactualizó en el ecosistema digital, aunque surgió durante el apogeo de los medios masivos, cuando la producción y la circulación de noticias obedecía al modelo heredado de la Revolución Industrial (Hirst, 2011), es la de los usos y gratificaciones (Katz et al., 1974). Desde sus orígenes, este corpus de conocimiento puso el acento en las motivaciones del público para elegir determinados medios con el objetivo de informarse, conseguir diversión, compañía, notoriedad o conocimientos (Moragas Spà, 2003).

Desde esta perspectiva, Igartua et al. (2020), en un estudio cuantitativo a través de encuestas en España, pudieron determinar el modo en el que una plataforma mediática -WhatsApp - tuvo un uso prioritario durante el confinamiento, en relación con redes sociales y medios tradicionales, por ejemplo, para consumo informativo. García-Ruiz et al. (2018) hicieron una investigación cuantitativa - a través de encuestas en donde se aplicaron escalas de Likert- respecto al uso de redes sociales por parte de estudiantes de educación secundaria y universitaria en España. Los resultados preliminares indican que los alumnos de educación superior, en las redes, buscan principalmente entretenimiento.

Nyembe y Howard (2020) han analizado el uso de WhatsApp como parte de las estrategias de aprendizaje colaborativo que llevan adelante los estudiantes, desde la perspectiva del aprendizaje móvil colaborativo ( $\mathrm{MCL}$, por sus siglas en inglés) y de la teoría del aprendizaje móvil (Kearney et al., 2012; Sharples et al., 2005). Para estos autores, WhatsApp tiene potencial para promover la interacción y la participación durante las actividades de aprendizaje, habilita el aprendizaje colaborativo asincrónico y ayuda a crear inmediatez y conexión en el aprendizaje formal e informal (Nyembe y Howard, 2020). En la misma línea, el trabajo de revisión documental de Montenegro (2020) sostiene que WhatsApp ha adquirido importancia significativa en el campo de la educación superior universitaria con un impulso notable al desarrollo del aprendizaje colaborativo entre estudiantes y docentes.

Un trabajo de Carcelén et al. (2019), realizado en España sobre la percepción de los estudiantes universitarios acerca del uso del móvil en su tiempo de aprendizaje y de las posibles repercusiones en su rendimiento académico, ha concluido que, más allá de las diferencias en cuanto al tipo de estudios elegido o la universidad en la que se cursan, un factor importante es el grado de autocontrol y responsabilidad manifestado por el propio alumno acerca del uso del móvil durante el tiempo de aprendizaje. Según dicho estudio, hay estudiantes más permisivos con el uso de las TIC en las aulas, que creen que su utilización no afecta de forma negativa en sus estudios; y otros más conscientes y responsables, que desarrollan mecanismos de autocontrol para limitar el uso del móvil tanto en clase como cuando estudian (Carcelén et al., 2019).

Por otra parte, en Argentina, un trabajo de la Universidad Nacional de Córdoba, que indagó sobre las condiciones de acceso tecnológico por parte de estudiantes secundarios y 
universitarios de esa provincia en el marco de esta cuarentena obligatoria por la pandemia del COVID-19, reveló que el dispositivo más utilizado por los estudiantes es el teléfono celular $(86,6 \%)$, seguido de los notebooks o netbooks (57,7 \%) y las computadoras de escritorio (43,8\%). Asimismo, el estudio afirma que "el alto uso del celular en los estudios apunta a un uso complementario de otros dispositivos como notebooks, netbooks o PC" (Ardini et al., 2020). Asimismo, una encuesta sobre las condiciones de conectividad de estudiantes universitarios de la provincia de Buenos Aires, realizada en el 2020 por el Consejo Provincial de Coordinación con el Sistema Universitario y Científico de la provincia de Buenos Aires (Argentina) y el Ente Nacional de Comunicaciones arrojó como resultados que el $32,86 \%$ de los estudiantes utilizan más de un dispositivo para sus tareas y clases virtuales, mientras que de los que se valen de un solo dispositivo, el $30,51 \%$, emplea solo la notebook; el $24,45 \%$ solo el teléfono móvil, el $11,45 \%$ solo computadora de escritorio y, en último lugar, solo usa tablet el $0,74 \%$ de los casos.

\section{METODOLOGÍA}

Esta investigación cualitativa es de alcance descriptivo², ya que "busca especificar propiedades, características y rasgos importantes de cualquier fenómeno que se analice. Describe tendencias de un grupo o población" (Hernández Sampieri et al., 2010, p. 80).

La técnica de recolección de datos fue la entrevista en profundidad, que, de acuerdo con Valles (1997), tiene diversos significados. Sin embargo, para los fines de esta investigación, el concepto se ciñe al de entrevista focalizada. En cuanto a las ventajas del uso de las entrevistas en profundidad, Valles (1997) destaca que "el estilo especialmente abierto de esta técnica - al igual que otras técnicas cualitativas - permite la obtención de una gran riqueza informativa" (p. 196). Para Taylor y Bogdan (1994), las entrevistas en profundidad tienen las mismas características que un diálogo entre iguales, y no como un intercambio formal de preguntas y respuestas, en donde el investigador quiere "esclarecer experiencia humana subjetiva" (p. 106).

La muestra está compuesta por 45 informantes, de entre 18 y 24 años, que estudian en universidades argentinas públicas y privadas ubicadas en la Ciudad Autónoma de Buenos Aires y en el conurbano bonaerense por una cuestión de factibilidad y de accesibilidad de los investigadores. Para la selección de casos se combinó el tipo de muestreo de "construcción teórica" - la selección se hace sobre informantes que se consideran a priori ricos en información sobre el tema que se está investigando- con el muestreo

2 La presente investigación fue realizada con el patrocinio de la Facultad de Ciencias de la Educación y la Comunicación Social de la Universidad del Salvador en el bienio 2019-2020, bajo el título “La influencia de la tactilidad en el uso de los dispositivos móviles para el estudio por parte de jóvenes universitarios". 
"bola de nieve", según el cual un informante nos va recomendando a otro, mediante el uso de redes de relaciones preexistentes (Lindlof, 1995). En este sentido, se partió de una base mínima de 15 casos, pero se dejaron de hacer entrevistas al llegar al número 45, de acuerdo con el criterio de saturación de la muestra, porque no se encontraron variables significativas o patrones nuevos en los entrevistados (Valles, 1997).

El análisis de los resultados cualitativos se realizó a través del "análisis temático" (Escudero, 2020), un tipo de análisis centrado en los temas y subtemas que emergen de los dichos de los entrevistados (Valles, 1997). Esto implicó un profundo contacto con los datos recolectados para asociar fragmentos de entrevistas a determinadas categorías que surgieron del trabajo con el corpus a través del programa informático Atlas.ti (versión 8.3.1). A partir de dicho análisis temático, se creó una serie de códigos en los que se organizaron las citas más significativas.

\section{RESULTADOS}

Para la presentación de los resultados de la investigación en este artículo, se han seleccionado dos códigos que vinculan distintos fragmentos obtenidos durante las entrevistas a los informantes que componen la muestra. El primero se centra en el uso del smartphone por parte de los estudiantes en su vida cotidiana, excepto en lo relacionado con el estudio, y el segundo código se refiere específicamente al uso de los dispositivos móviles inteligentes para estudiar.

En cuanto a la presentación de las entrevistas, cabe aclarar que hemos optado por ilustrar las conceptualizaciones de nuestro análisis con citas de los entrevistados, acompañadas de su nombre de pila - para preservar el anonimato-, la edad y la carrera que cursan. No es objeto de esta investigación hacer comparaciones o ponderaciones sobre el tipo de carrera que han elegido y su correlato con los usos que desempeñan. Solo se indican estos datos a fin de tener una idea de la heterogeneidad de la muestra y de la transversalidad que tienen estos usos en los estudiantes universitarios.

\section{El smartphone en la vida cotidiana de los estudiantes}

En este apartado los estudiantes entrevistados cuentan para qué usan el dispositivo móvil inteligente. Interesa, en esta parte de la investigación, detenerse en las funciones que cumple el smartphone; por lo tanto, se les preguntó a ellos qué uso le dan en su vida cotidiana y las actividades que realizan con sus teléfonos.

A veces la uso para sacar fotos. A veces, si me junto con un amigo, que a veces nos juntamos para hacer música o algo, lo usamos para grabar alguna "zappada" que estemos haciendo, alguna idea que esté buena. (Nicolás, 23 años, Informática)

El celular lo uso para lo que sea porque es más práctico, por ejemplo, para redes 
sociales, ver cosas, y la computadora, uso Spotify para dejar la música de fondo. (Augusto, 22 años, Comunicación)

Habitualmente uso Instagram, WhatsApp. WhatsApp tanto para la facultad como para comunicarme con amigas. Tenemos muchos grupos de la facultad [...] uso mucho Spotify para escuchar música, uso YouTube tanto para aprender como para divertirme. (Carolina, 24 años, Ciencias Biológicas)

Más allá del consumo de contenidos audiovisuales, muchos estudiantes utilizan los smartphones para intervenir las imágenes o editarlas.

Edición de fotos PhotoGrid y Pixart. Y videos InShot, PicPlayPost. Aprendí a usarlo sola, son bastante intuitivas. (María, 23 años, Psicología)

Más que nada lo uso para las redes sociales, WhatsApp y escuchar música. (Martina, 19 años, Diseño Gráfico)

Editar fotos, trabajo, tomar notas, estudio, ocio, mirar videos. Aprender. Aprendo un montón con el celular. Esta cuestión de los algoritmos me permite aprender cosas nuevas a partir de las propuestas. (Dante, 21 años, Relaciones Públicas)

En el siguiente extracto, uno de los informantes refiere todas las aplicaciones que utiliza en el celular e incluso su combinación con el consumo de medios tradicionales:

El celular lo uso para lo que sea porque es más práctico, por ejemplo, para redes sociales, ver cosas, y la computadora uso Spotify para dejar la música de fondo. En otro momento, si quizás estoy viendo algún programa de la televisión y quiero saber lo que está opinando la gente en ese momento, uso el teléfono para ver las redes, por ejemplo, Twitter. (Augusto, 20 años, Comunicación)

En suma, el smartphone desempeña múltiples funciones en la vida cotidiana de los jóvenes, quienes encontraron en la cuarentena la posibilidad de explorar nuevas prestaciones y extenderlo a más actividades. En línea con otras investigaciones consignadas en este texto, los informantes dieron cuenta de un cambio a partir del aislamiento social provocado por la pandemia del COVID-19, que implicó la utilización más intensiva de la computadora de escritorio, la netbook o la notebook en detrimento del teléfono celular inteligente.

\section{El smartphone para estudiar en grupo}

Este apartado reúne todas aquellas manifestaciones respecto del uso del smartphone para prácticas académicas. Varios estudiantes hicieron referencia al trabajo colaborativo gracias a sistemas de mensajería instantánea, sobre todo al momento de preparar un examen parcial o resolver un ejercicio. 
Slack está bueno porque podemos así aportar conocimientos entre todos y cada tanto me bajo alguna que otra imagen o libro que comparten que están muy buenos. (Francisco, 24 años, Diseño Industrial)

También la comunicación con mis compañeros, que es como gran parte de todo lo que es la parte del estudio, digamos, todo lo que tiene que ver con hacer trabajos, parciales, fechas importantes, leíste tal texto, todo eso lo manejo por WhatsApp de manera más informal. (Alejandro, 21 años, Educación)

El trabajo colaborativo entre los estudiantes se ve plasmado, sobre todo, en la posibilidad que brindan los sistemas de mensajería de crear grupos. Así, los informantes aseguran que se reúnen en esos espacios vinculados por el cursado de una materia en común, a pesar de que a veces ni siquiera han llegado a conocerse en persona.

Uso mucho WhatsApp, creo que no lo uso excesivamente, me sirve mucho para la facultad, para organizarme con la gente, para charlar [...] Llegamos a hacer grupos de WhatsApp de 100 personas, con gente con la que cursamos la materia que capaz ni conocíamos y más que nada para los finales, lo organizamos para poder estudiar, para rendir y lo que hicimos fue organizarnos, como mandar ejercicios resueltos, o ir preguntando dudas por ahí, sirve un montón. (Victoria, 20 años, Ingeniería Ambiental)

En WhatsApp yo tengo un grupo con unos compañeros de la facultad que por lo general siempre pasan links para unos trabajos de la facultad que tenemos que hacer en grupos. Dentro de lo académico usamos mucho WhatsApp. Ya ahí nos sacamos dudas de encima con respecto a un examen o vamos tirando ideas para un trabajo práctico, realmente lo explotamos mucho en ese sentido. (Francisco, 21 años, Inglés)

Tenemos muchos grupos de la facultad, donde nos pasamos dudas, pasamos parciales o exámenes hechos, consultamos preguntas de las clases. Nos organizamos si queremos, a veces, hacer una queja general para la materia, si hay algún problema, primero lo discutimos todos los alumnos y después le mandamos un mail al profesor. (Carolina, 24 años, Biología)

El celular, la única función relacionada al estudio que le doy, es si tengo que hablar con compañeros para hacer algún tipo de repaso o para preguntar por alguna cuestión, algún tema que pueda no saber, más bien en lo que es comunicación. (Ignacio, 18 años, Derecho)

\section{El smartphone y los apuntes orales}

Otro uso cada vez más extendido tiene que ver con la posibilidad de las plataformas de mediatizar el sonido. Varios de los entrevistados manifestaron que utilizan la grabación de audios para repetir conceptos o hacer algunos "apuntes orales" que luego pueden ir reproduciendo en situaciones de movilidad (por ejemplo, viajando hacia la facultad o hacia el trabajo), en las que no pueden recuperar textos escritos. Debe destacarse que el uso de la mediatización del sonido por parte de nuestros informantes combina lo puramente 
individual y lo grupal: es decir, aparecen audios que no se comparten en los grupos colaborativos mencionados más arriba y otros que sí. En muchas ocasiones, las notas de voz se utilizan para estudiar y se vinculan a una actividad cognitiva: los entrevistados estudian al preparar su "apunte oral" y luego "repasan" cuando los oyen una y otra vez.

Todo el tiempo. Uso mucho el dictado de voz. Todo el tiempo. Todo lo que va diciendo el profesor yo lo replico con mis palabras y lo transcribo. (Sebastián, 18 años, Periodismo)

Cuando termino de repasar y quiero volver a repasar me grabo un audio con todo con el celular diciendo lo que sé. Me gusta después escuchar los audios y como que me entra más información yendo a la facultad, me escucho a mí hablando. (Camila, 24 años, Periodismo)

Me grabo audios para escucharlos, me sirve muchísimo. 0, si tengo que leer algún archivo o algo, de vez en cuando también hago grabaciones. Estoy en mi casa y grabo algo que tengo en papel y, cuando estoy en el colectivo, lo escucho para estudiarlo. (Joaquín, 18 años, Derecho)

La especificidad técnica de alguna disciplina que involucra la fonética (por ejemplo, la carrera de Traductorado de Inglés) puede hacer que los estudiantes compartan audios para mejorar o corregir su pronunciación.

En realidad usamos mensajes de voz como para aclararle a algún compañero de los mensajes de audio, el mensaje de voz: de vez en cuando se utilizan, más que nada para una materia que se llamaba Fonética, en la que nos grabamos, escuchábamos el audio después y veíamos qué onda la pronunciación. (Francisco, 21 años, Inglés)

Los audios de WhatsApp determinan también aspectos de trabajo colaborativo, en tanto y en cuanto un estudiante plantea una pregunta "de parcial" en el grupo y el resto da su opinión a través de notas de voz. Inclusive, puede afirmarse que este tipo de audios también cumple una función pedagógica, dado que sirven para que un alumno pueda resolver dudas o preguntas de otros estudiantes sobre un tema.

Cuando tengo que rendir un examen, nos preparamos a veces con amigos, preguntas típicas de parcial, y las vamos contestando por nota de voz, o cuando alguien no entendió un tema y me pide que lo explique; a mí me sirve explicar para estudiar, más tomando notas de voz. (Carolina, 24 años, Biología)

\section{El smartphone y el acceso a los textos}

Las características portables y multimedia del smartphone permiten que los estudiantes universitarios puedan acceder a contenidos bibliográficos y textuales desde el propio dispositivo. En este caso, con el teléfono celular inteligente pueden hacer capturas de pantalla sobre partes relevantes o intervenir de alguna manera el texto que leen. En 
algún caso, el celular funciona también como un reemplazo de los apuntes en papel, que son valorados, pero que se dejan de lado por una cuestión de costos.

Descargo archivos ahí y los puedo leer. Puedo resaltarlos para sacar partes clave, y puedo sacar capturas de información que encuentre paralela. (Camila, 23 años, Periodismo)

Uso el celular para leer los [archivos] Word y los mails, y para los PDF que me mandan. (Martina, 19 años, Diseño Gráfico)

Cuando tengo que estudiar son archivos de muchas páginas, que no los pude imprimir o es muy costoso, los leo del celular. (Damián, 20 años, Ingeniería Industrial)

Otra de las actividades que aparecen vinculadas al estudio a través del smartphone es la búsqueda. La posibilidad de tener cerca el dispositivo móvil hace que sirva para hacer búsquedas mientras se aborda un texto, o para consultar alguna duda mientras se escucha una clase (presencial o sincrónica virtual), o lo que los profesores puedan llegar a mencionar en ella.

Estudio con el teléfono. Siempre. Busco muchas cosas con el teléfono. 0 sea, no solamente para el estudio, sino cosas del laburo. Para el estudio busco significados, portales, páginas que hayan recomendado en Twitter, podcast. (Camila, 24 años, Relaciones Públicas)

Lo uso mucho para buscar cosas: estoy leyendo algo y hay un concepto que no sé; o hay algo que... sobre todo, como escribo mucho, por ahí tengo que buscar sinónimos o estas cuestiones y ahí también lo uso. (Juliana, 23 años, Comunicación)

Si estoy cursando alguna materia y no tengo la computadora cerca, lo uso para buscar información o completar alguna cosa que falte respecto al tema que estamos viendo. (Isabella, 21 años, Dirección de Comunicaciones)

Cuando algún profesor nombra un autor o un libro, lo busco, le hago una captura a la pantalla y lo dejo en la galería de fotos para después buscar información. (Luna, 18 años, Relaciones Públicas)

En algún caso, se destaca la búsqueda como recurso didáctico de los profesores que incentivan esa actividad en el transcurso de una clase presencial o un encuentro sincrónico.

Hay algunos profesores que nos incentivan como para buscar información bibliográfica sobre algún autor en particular cuando tenemos que ver algo más sobre comprensión lectora. (Francisco, 21 años, Inglés)

\section{El smartphone y el uso organizativo}

El acceso a aplicativos institucionales de las universidades en donde estudian nuestros informantes es otro de los usos más frecuentes del smartphone en relación con 
las actividades académicas. Los recursos mencionados son plataformas virtuales (que actúan como repositorios para las clases) o también aplicativos específicos que funcionan como sistema de mensajería, anuncios y cartelería virtual (se publican, por ejemplo, en qué aulas son las cursadas, las fechas de las mesas de exámenes, los cronogramas de clases). Por el advenimiento de la pandemia del COVID-19 y el desarrollo de clases a distancia, varios entrevistados nos refirieron que las universidades en las que estudian recrearon nuevos entornos virtuales de enseñanza y aprendizaje a través de foros y espacios de consulta a los que se puede acceder a través del teléfono celular.

Tenemos en la universidad una plataforma a la que tenés que acceder por internet. Podés entrar por una computadora o cualquier dispositivo. Yo, generalmente, uso el celular o la notebook. Pero con el celular lo que hago es bajarme siempre todas las cosas, porque ellos archivo que te dan, archivo que te hacen bajar. (Damián, 20 años, Ingeniería Industrial).

Uso la aplicación de la facultad para saber las aulas en las que curso y las fechas de finales, leo los blogs de los profesores para ver ejemplos de trabajos. (Luna, 18 años, Relaciones Públicas)

Tengo en el teléfono instalado Google Drive, Google Docs y tengo el campus de la UBA. (Florencia, 19 años, Medicina)

Tengo la aplicación de la USAL, para inscripción y faltas, aunque no falto mucho, trámites administrativos. (Luciana, 20 años, Diseño Gráfico)

Usamos mucho el Campus Virtual, en donde la facultad creó distintos foros, y los estamos usando muchísimo. En el foro los profesores pueden ver nuestras preguntas, pero otros alumnos también y se pueden subir imágenes o archivos y vos, entonces, vos hacés una pregunta y cualquiera te puede contestar y te puede dar un feedback, te puede decir qué está mal, te puede explicar, está muy bueno. (Carolina, 24 años, Biología)

Otra de las funciones que cumple el teléfono móvil inteligente respecto del estudio es el denominado "uso organizativo" (Carcelén et al., 2019), que está vinculado a aplicaciones de gestión de apuntes, del tiempo y del trabajo, los calendarios y el reloj.

Se destaca el uso de Google Drive porque permite una organización de apuntes y de notas, y se combina con la posibilidad de realizar trabajo colaborativo.

Tengo el calendario y recordatorios. También tengo la aplicación de la USAL, donde tengo todas las notas, eventos y cosas así. Classroom y Drive. (Sofía, 21 años, Diseño Gráfico)

Las herramientas de Google, que facilitan bastante, como Google Docs o Drive para trabajos colaborativos. Keep, de notas, que me permite anotar cosas de clase y lo que tengo pendiente hacer o las ideas que tengo. (Camila, 23 años, Periodismo)

Otra herramienta que los profesores también utilizan un montón es Google Drive porque a partir de eso, más que nada con las traducciones que tenemos que hacer, 
grupales, ya que todos podemos modificar el archivo, cada uno pone su parte. Además, tenemos la opción de poner comentarios o poner una duda y que otro compañero más adelante dé su opinión al respecto. Son las dos aplicaciones más utilizadas dentro del ámbito académico. (Francisco, 21 años, Inglés)

Las características multimediales y nómades del smartphone le permiten ser reconocido por nuestros entrevistados como un dispositivo con usos múltiples vinculados a las actividades académicas. Se destaca entre ellas la posibilidad del trabajo colaborativo, como resolver dudas, compartir apuntes, fotografías de pizarrones y socializar cualquier tipo de material que esté vinculado al estudio.

Por otro lado, poco a poco va ganando espacio la mediatización del sonido a través de la producción de pequeños "apuntes orales" que sirven para que los estudiantes puedan repasar antes de un examen o parcial en situaciones de traslado, en viajes o en momentos en los que no les es posible interactuar con un texto escrito.

Las posibilidades de búsqueda sincrónica se han incrementado gracias a la presencia ubicua del teléfono celular inteligente: esta práctica se hace ya sea en medio de la lectura concentrada de apuntes, sea por incentivo o indicación de un profesor, o bien por propia necesidad del estudiante, para contextualizar un tema o resolver una duda.

Muchas casas de estudio han desarrollado aplicativos institucionales no solo para las actividades académicas propiamente dichas (participación en clases, foros de consulta), sino también para cuestiones vinculadas con el registro administrativo (ubicación de clases, grillas de horarios y de cursadas, inscripción a exámenes finales, consulta de inasistencias), algo muy utilizado por los estudiantes desde el dispositivo móvil.

Por último, el "uso organizativo" también está presente debido a que el celular permite la instalación de diversas aplicaciones que sirven para gestionar el tiempo (calendarios, alarmas), así como también diversos materiales de estudio, como apuntes y bibliografía.

\section{DISCUSIÓN Y CONCLUSIONES}

El trabajo de campo de esta investigación revela el modo en el que las múltiples funciones que cumplen los dispositivos móviles en la vida cotidiana de los estudiantes universitarios fueron resignificadas a partir del aislamiento social provocado por la pandemia del COVID-19.

Estas transformaciones también afectaron el uso de otros sistemas de intercambio: desde una mirada ecológica, los medios o tecnologías de comunicación no pueden analizarse de manera aislada o separada, dado que cada uno de ellos aporta al ecosistema un conjunto de usos y de posibilidades, también asociados al resto. Esta cuestión se vincula 
al concepto de polymedia (Miller, 2016) que, desde la antropología, indica que los usos de medios y plataformas están relacionados entre sí. Varias investigaciones (Arri, 2020; Albarello, 2020a, 2020b, 2016) han dado cuenta del uso "cruzado", por parte de jóvenes estudiantes universitarios, de medios y plataformas en donde se hibridan contenidos y consumo.

Por lo general, la combinación de uso entre computadora y teléfono móvil inteligente está supeditada a las funcionalidades. El smartphone, más asociado al esparcimiento, al uso de redes sociales, visionado de videos y entretenimiento, permite, en términos de Igarza (2009), la generación de "burbujas de ocio" de tipo intersticial. En tanto que la preferencia por la computadora ocurre en situaciones de estudio, trabajo y realización de actividades académicas. De todos modos, el uso cruzado también ocurrió en situación de estudio, puesto que algunos informantes mencionaron que mientras tomaban una clase virtual sincrónica en la computadora, hacían una búsqueda o tomaban una nota tipo apunte en el teléfono celular.

Los informantes dieron cuenta de la incidencia del smartphone para la gestión del trabajo colaborativo vinculado a actividades académicas durante el periodo de aislamiento. En este sentido, los grupos de WhatsApp crearon espacios de intercambio incluso con otros estudiantes "desconocidos": se constituyeron en usinas de ayuda ante determinadas situaciones, como exámenes parciales. Esta colaboración grupal se evidenció en las actividades relacionadas con el "compartir": audios con explicaciones, material de estudio (apuntes, bibliografía) o incluso explicaciones ante simulaciones de exámenes dentro de cada uno de los grupos.

En línea con los resultados de otras investigaciones, el diálogo con los informantes nos mostró que varios de ellos utilizan el smartphone en relación con el estudio logrando una "integración móvil" (Limón-Flores, 2018), es decir, generando distintos tipos de vínculos establecidos dentro de los centros de estudios para cumplir con actividades colaborativas, en el marco de lo que Jenkins (2008, p. 37) denomina "comunidades virtuales" que "se mantienen unidas mediante la producción mutua y el intercambio recíproco de conocimientos", lo que podemos asociar con la noción de "inteligencia interconectada", que para Tapscott (1998) no implica solamente un entrecruzamiento de tecnologías, sino la interconexión de personas a través de la tecnología, combinando su inteligencia a través de las redes.

En términos de Miller (2016), el uso de cada plataforma propone distintos grados o escalas de sociabilidad, lo que permite explicar el uso individual/grupal de un sistema de mensajería como WhatsApp para las prácticas académicas. Los sistemas y las posibilidades de intercambio en enormes grupos entre estudiantes que nunca se conocieron de manera presencial, pero que cursan un mismo espacio curricular, puede ser definido en términos de un aspecto más de la "socialidad por plataformas" (Van Dijck, 2016). 
Debe resaltarse que, en algunos casos, los estudiantes también tuvieron que intensificar el uso del smartphone para tareas referidas al estudio, porque las universidades en donde cursan sus carreras desarrollaron aplicativos institucionales no solo para la gestión académica (acceso a campus virtual, recursos en línea), sino también para cuestiones vinculadas con el plano administrativo (solicitud de certificaciones, chequeo de asistencia virtual). Dentro de este "uso organizativo" del smartphone (Carcelén et al., 2019), se menciona el uso de aplicaciones de la "nube" (Google Drive, Dropbox) que permiten a los alumnos organizar el material de estudio (bibliografía y apuntes) y recuperarlos desde el dispositivo mientras, por ejemplo, cumplen con otra tarea en la computadora.

Los hallazgos aquí presentados permiten concluir que el uso que hacen del smartphone los estudiantes universitarios, en el marco particular que impone la pandemia del COVID-19, está totalmente asociado a su experiencia académica, la cual asume un rasgo marcadamente colaborativo por las posibilidades comunicativas que habilita este dispositivo metamedium en su vida cotidiana.

\section{REFERENCIAS}

Aguado, J. M., Feijóo, C., y Martínez, I. (2013). La comunicación móvil. Hacia un nuevo sistema digital. Gedisa.

Albarello, F. (2016). El ecosistema digital de los jóvenes universitarios: una mirada desde la Ecología de los medios. En J. C. Durand, Á. V. Corengia, F. T. Daura (Dirs.), y M. S. Urrutia (Coord.), Aprender a enseñar: el desafío de la formación docente inicial y continua (pp. 115-136). TeseoPress. https://www.teseopress.com/ formaciondocente/chapter/183/

Albarello, F. (2020a). De la hegemonía al nicho: desplazamiento de medios tradicionales en rutinas informativas de un grupo de universitarios argentinos. Dixit, 32, 46-60.

Albarello, F. (2020b). Informarse en el smartphone: estrategias de lectura transmedia por parte de jóvenes universitarios del Aglomerado Gran Buenos Aires. Palabra Clave, 23(3), e2331.

Albarello, F., y Kelly, V. (2019). Multimodalidad y ecología de los medios: aproximaciones conceptuales para repensar los medios en el aula. 1.er Congreso Internacional de Ciencias Humanas - Humanidades entre pasado y futuro. Escuela de Humanidades, Universidad Nacional de San Martín, Gral. San Martín. https://www.aacademica. org/1.congreso.internacional.de.ciencias.humanas/1103.pdf

Arri, F. (2020). Leer en smartphones. Estrategias de lectura/navegación de estudiantes universitarios de carreras vinculadas a la Comunicación en teléfonos móviles [Tesis doctoral, Universidad Austral]. https://riu.austral.edu.ar/bitstream/ 
handle/123456789/941/Tesis\%20Doctoral\%20Francisco\%20Arri-Version\%20 Final.pdf?sequence $=1$

Ardini, C., Barroso, M. B., Contreras, L., y Corzo, L. (2020). Estudiar durante una pandemia: una mirada al rol del estudiante y la experiencia educativa en entornos virtuales en el marco de la pandemia COVID-19. Mutual Conexión; Universidad Nacional de Córdoba, Facultad de Ciencias de la Comunicación. https://rdu.unc.edu.ar/ handle/11086/15888

Aufa, A., Mustansyir, R., y Maharani, S. (2020). What is Smartphone? Philosophical Perspective: Reflection on the Use of Smartphone in the New Normal Life. Research, Society and Development, 9(9), e116996991. http://doi.org10.33448/ rsd-v9i9.6991

Baert, S., Vujić, S., Amez, S., Claeskens, M., Daman, T., Maeckelberghe, A., Omey, E., y De Marez, L. (2020). Smartphone Use and Academic Performance: Correlation or Causal Relationship? Kyklos, 73, 22-46. https://doi.org/10.1111/kykl.12214

Bolter, J. (2001). Writing Space: The Computer, Hypertext, and the Remediation of Print. Lawrence Erlbaum Assoc.

Bruning, R. H., Schraw, G. J., Norby, M. M., y Ronning, R. R. (2004). Cognitive Psychology and Instruction (4. ${ }^{\text {a }}$ ed.). Pearson.

Carcelén, S., Mera, M., e Irisarri, J. A. (2019). El uso del móvil entre los universitarios madrileños: una tipología en función de su gestión durante el tiempo de aprendizaje. Communication \& Society, 32(1), 199-211.

Castells, M. (2009). Comunicación y poder. Alianza Editorial.

Chuchu, T., y Ndoro, T. (2019). An Examination of the Determinants of the Adoption of Mobile Applications as Learning Tools for Higher Education Students. International Journal of Interactive Mobile Technologies (IJIM), 13(03), 53-67. http:// dx.doi.org/10.3991/ijim.v13i03.10195

Consejo Provincial de Coordinación con el Sistema Universitario y Científico de la provincia de Buenos Aires; Ente Nacional de Comunicaciones. (2020). Resultados encuesta sobre condiciones de conectividad en estudiantes universitarios de la provincia de Buenos Aires. https://tinyurl.com/kv2t9ep8

Cote-Parra, G. E. (2015). Engaging Foreign Language Learners in a Web 2.0-Mediated Collaborative Learning Process. Profile: Issues in Teachers' Professional Development, 17(2), 137-146. http://dx.doi.org/10.15446/profile.v17n2.47510

Dafonte-Gómez, A., Maina, M. F., y García-Crespo, 0. (2021). Uso del smartphone en jóvenes universitarios: una oportunidad para el aprendizaje. Píxel-Bit. Revista de Medios y Educación, 60, 211-227. https://doi.org/10.12795/pixelbit.76861 
Davis, F. D., Bagozzi, R. P., y Warshaw, P. R. (1989). User Acceptance of Computer Technology: A Comparison of Two Theoretical Models. Management Science, 35(8), 982-1003. https://doi.org/10.1287/mnsc.35.8.982

Derogan la resolución que prohibía los celulares para uso pedagógico. (27 de octubre del 2016). Telam. https://tinyurl.com/pxjk34d2

Fidler, R. (1998). Mediamorfosis. Comprender los nuevos medios. Granica.

Escudero, C. (2020). El análisis temático como herramienta de investigación en el área de la Comunicación Social: contribuciones y limitaciones. La Trama de la Comunicación, 24(2), 89-100. https://doi.org/10.35305/lt.v24i2.746

Fernández, J. L. (2018). Plataformas mediáticas. Elementos de análisis y diseño de nuevas experiencias. La Crujía.

Fernández, J. L. (2021). Vidas mediáticas. Entre lo masivo y lo individual. La Crujía.

Flórez Buitrago, L., Ramírez García, C., y Ramírez García, S. (2016). Las TIC como herramientas de inclusión social. 3C TIC. Cuadernos de Desarrollo Aplicados a las TIC, 5(1), 54-67. https://doi.org/10.17993/3ctic.2016.51.54-67

García-Ruiz, R., Tirado Morueta, R., y Hernando Gómez, Á. (2018). Redes sociales y estudiantes: motivos de uso y gratificaciones. Evidencias para el aprendizaje. Aula Abierta, 47(3), 291-298. https://doi.org/10.17811/rifie.47.3.2018.291-298

Hernández Sampieri, R., Fernández-Collado, C., y Baptista, L. (2010). Metodología de la investigación. McGraw-Hill.

Hirst, M. (2011). News 2.0: Can Journalism Survive the Internet? Allen \& Unwin.

Igartua, J. J., Ortega-Mohedano, F., y Arcila-Calderón, C. (2020). Communication Use in the Times of the Coronavirus. A Cross-Cultural Study. El Profesional de la Información, 29(3), e290318. https://doi.org/10.3145/epi.2020.may.18

Igarza, R. (2009). Burbujas de ocio. Nuevas formas de consumo cultural. La Crujía.

Instituto Nacional de Estadísticas y Censos. (2019). Encuesta Permanente de Hogares. Módulo de Acceso y Uso de Tecnologías de la Información y la Comunicación. https:// bit.ly/3jKvNnk

Jenkins, H. (2008). Convergence Culture. La cultura de la convergencia de los medios de comunicación. Paidós.

Katz, E., Blumler, J. G., y Gurevitch, M. (1974). Utilization of Mass Communication by the Individual. En J. G. Blumler y E. Katz (Eds.), The Uses of Mass Communications: Current Perspectives on Gratifications Research (pp. 19-31). Sage. 
Kearney, M., Schuck, S., Burden, K., y Aubusson, P. (2012). Viewing Mobile Learning from a Pedagogical Perspective. Research in Learning Technology, 20, 1-17. https://doi. org/10.3402/rlt.v20i0.14406

Kress, G. (2005). El alfabetismo en la era de los nuevos medios de comunicación. Ediciones El Aljibe; Enseñanza Abierta de Andalucía.

Limón-Flores, C. A. (2018). Uso de dispositivos móviles de comunicación por estudiantes universitarios en el Instituto Tecnológico y de Estudios Superiores de Monterrey (ITESM), campus Guadalajara. RECIE. Revista Electrónica Científica de Investigación Educativa, 4(1), 709-720. https://bit.ly/3ywCwaR

Lindlof, T. (1995). Qualitative Communication Research Methods. Sage.

Manovich, L. (2013). Software Takes Command. Bloomsbury Academic.

Márquez, I. (2015). Una genealogía de la pantalla. Del cine al teléfono móvil. Anagrama.

Márquez, I. (2017). El smartphone como metamedio. Observatorio, 11(2), 61-71. https://doi. org/10.15847/obsOBS11220171033

McLuhan, M. (1994). Comprender los medios de comunicación. Las extensiones del ser humano. Paidós.

Miller, D. (2016). How the World Changed Social Media. University College of London Press.

Montenegro, D. (2020). Comunicación grupal en WhatsApp para el aprendizaje colaborativo en la coyuntura COVID-19. Hamut'ay, 7(2), 34-45.

Moragas Spà, M. (2003). Interpretar la comunicación. Estudios sobre medios en América y Europa. Gedisa.

Morán, L. (2021). Prácticas evaluativas en contextos de aula invertida y aprendizaje móvil. Innovaciones Educativas, 23(34), 98-112. https://doi.org/10.22458/ie.v23i34.3152

Mustapha, I., Thuy Van, N., Shahverdi, M., Qureshi, M., y Khan, N. (2021). Effectiveness of Digital Technology in Education during COVID-19 Pandemic. A Bibliometric Analysis. International Journal of Interactive Mobile Technologies (IJIM), 15(8), 136-154. http://dx.doi.org/10.3991/ijim.v15i08.20415

Nyembe B. Z. M., y Howard G. R. (2020). Development of a Quantitative Instrument to Measure Mobile Collaborative Learning (MCL) Using WhatsApp: The Conceptual Steps. En M. Hattingh, M. Matthee, H. Smuts, I. Pappas, Y. Dwivedi, y M. Mäntymäki (Eds.), Responsible Design, Implementation and Use of Information and Communication Technology. I3E 2020. Lecture Notes in Computer Science, 12066 (pp. 507-519). Springer. https://doi.org/10.1007/978-3-030-44999-5_42

Postman, N. (1985). Amusing Ourselves to Death. Penguin. 
Rojas-Osorio, M., y Álvarez-Risco, A. (2019). Intention to Use Smartphones among Peruvian University Students. International Journal of Interactive Mobile Technologies (IJIM), 13(3), 40-52. http://dx.doi.org/10.3991/ijim.v13i03.9356

Scolari, C. A. (2004). Hacer clic. Hacia una sociosemiótica de las interacciones digitales. Gedisa.

Scolari, C. A. (2012). Media Ecology: Exploring the Metaphor to Expand the Theory. International Communication Association, 22, 204-225. https://doi. org/10.1111/j.1468-2885.2012.01404.x

Scolari, C. A. (Ed.). (2015). Ecología de los medios. Entornos, evoluciones e interpretaciones. Gedisa.

Scolari, C. A. (2018). Las leyes de la interfaz: diseño, ecología, evolución, tecnología. Gedisa.

Sharples, M., Taylor, J., y Vavoula, G. (2005). Towards a Theory of Mobile Learning. En Proceedings of mLearn2005 - 4th World Conference on mLearning (pp. 1-9). Academic Press.

Silva Calpa, A., y Martínez Delgado, D. (2017). Influencia del smartphone en los procesos de aprendizaje y de enseñanza. Suma de Negocios, 8, 11-18. https://doi.org/10.1016/j. sumneg.2017.01.001

Srnicek, N. (2018). Capitalismo de plataformas. Caja Negra Editora.

Tapscott, D. (1998). Creciendo en un entorno digital. La generación net. McGraw-Hill.

Taylor, S. J., y Bogdan, R. (1994). Introducción a los métodos cualitativos en investigación. Paidós.

Valles, M. (1997). Técnicas cualitativas de investigación social. Reflexión metodológica y práctica profesional. Síntesis.

Van Dijck, J. (2016). La cultura de la conectividad. Una historia crítica de las redes sociales. Siglo XXI Editores. 
